(C)2007 IEEE. Personal use of this material is permitted. However, permission to reprint/republish this material for advertising or promotional purposes or for creating new collective works for resale or redistribution to servers or lists, or to reuse any copyrighted component of this work in other works must be obtained from the IEEE 


\title{
Contemporary Cultural Researches on Knowledge Sharing
}

\author{
Hai Dong, Farookh Khadeer Hussain, Elizabeth Chang \\ Digital Ecosystem and Business Intelligence Institute \\ Curtin University of Technology \\ Perth, Australia \\ Email: \{hai.dong, farookh.hussain, elizabeth.chang\}@cbs.curtin.edu.au
}

\begin{abstract}
Knowledge sharing, as an essential component in the field of knowledge management research, attracts increasing researchers into this discipline. Current researches on KS can be categorized as two independent views - exploring from technical perspective or from cultural perspective. From technical perspective, researchers studied multiple methodologies implementing KS; from cultural perspective, scholars studied social issues which influence the style and the performance of KS. In this paper, we propose to compare and contrast the contemporary cultural researches on knowledge sharing from multiple perspectives. Conclusion and future work are made in the final section.
\end{abstract}

\section{INTRODUCTION}

Knowledge sharing, as an essential component in the field of knowledge management research, attracts increasing researchers into this discipline. It is well known that the most valuable knowledge within an organization is not limited to the formal documents in the databases and repositories, but also includes the undocumented ideas, insights and know-how of its members [1]. However, this informal and tacit knowledge is deeply rooted in the individual experience and the culture of work community. To solve these problems, many methodologies have been proposed, which aims to exploit the embodied knowledge in practice and transform them to a normal form so that other staffs and organizations can utilize it. $\mathrm{KS}$ is one of these methods to solve the above problems.

Current researches on KS can be divided into two aspects technical and cultural. From technical perspective, researchers studied multiple methodologies implementing $\mathrm{KS}$; from cultural perspective, scholars studied social issues, which influence the style and the performance of $\mathrm{KS}$, such as ethnic culture [8], subjective norms, attitude and perceived behavioral control [10].

In this paper, we propose to compare and contrast the contemporary cultural researches on $\mathrm{KS}$. The literature of $\mathrm{KS}$ with regards to definitions and benefits are reviewed in Section 2. In Section 3 we analyze the cultural researches on $\mathrm{KS}$ from multiple perspectives. The conclusion and future work are made in Section 4.

\section{KNOWLEDGE SHARING LITERATURE REVIEW}

Recently many researchers start to recognize the importance defining $\mathrm{KS}$ and thus $\mathrm{KS}$ has been defined theoretically. In 2003, Cummings stated that KS involves 'the process through which knowledge is channeled between a source and a recipient' and its meaning within an organization is 'an organization that obtains access to its own and other organization's knowledge' [3]. In the same year, Soekijad and Andriessen considered $\mathrm{KS}$ involves the processes of knowledge creation, knowledge transfer and knowledge distribution [4]. Ryu et al. defined KS as "the behavior of disseminating ones acquired knowledge with other members within one's organization" [5]. In 2006, Jones et al. defined KS as "the sharing of knowledge about business processes and the related knowledge required making these processes work" [2]. Here I present the definition of $\mathrm{KS}$ - the process of translating the personalized knowledge into the collective knowledge, which consists of the processes of knowledge discovery, knowledge extraction and knowledge transfer. Following that, the main activities of $\mathrm{KS}$ are described. Here Cummings summarized three types of main $\mathrm{KS}$ activities, including those focused on 'accessing the form and embeddedness of the knowledge', those focused on 'establishing and managing an administrative structure through which differences and issues between the parties can be accommodated and reduced' and those focused on 'transferring knowledge' [3].

Then why use KS? From UTS's webpage (http:/linus.socs.uts.edu.au/), we can identify the most common quoted advantages for $\mathrm{KS}$. The first benefit is that $\mathrm{KS}$ can create 'newer and better products and services', which means 'ways of combining information are evaluated to see if new products and services can be easily produced'. The second benefit is experience transfer, which the experiential knowledge is the 'information stored as to how individual tasks were done in the past'. The following advantage is organizational learning, which whole organization is able to learn about the information that is kept about 'how things were done in the past'. The final advantage is transactional knowledge that is about 'where knowledge is'. Without the capacity for sharing knowledge, no business organizations can utilize the specialized resources and capabilities of its members, nor can it co-produce new knowledge. Thus, KS is a critical factor in terms of its relative competitiveness. 


\section{CULTURAL RESEARCHES ON KS}

In this section, we will introduce the researches on social issues, which influence the performance of $\mathrm{KS}$, and compare them from the perspective of research topic, domain, issues, solutions (if possible), advantages and limitations.

By a multi-site case study, Jones et al. analyses how cultural issues affects the $\mathrm{KS}$ in the process of ERP (Enterprise Resource Planning) implementation from eight aspects. By synthesizing the findings form the case study, the authors summarize a cultural configuration, which displays how the eight cultural aspects relate to KS. In addition, the authors configure out the initiatives to overcome the barriers in the eight cultural dimensions, in order to promote $\mathrm{KS}$ in ERP project organizations [2].

$\mathrm{Du}$ et al. propose to define the quantitative relationship between KS and its performance, by means of the mathematical deduction and survey method. The authors define six variables to measure $\mathrm{KS}$, and then propose the contingency models. Finally, the authors test the interrelationship of the six variables based on the survey data gathered from Xi' an, China, and the empirical result supports the authors' propositions [6].

Members in project organization are geographically dispersed and from different cultural background, which brings challenge to KS activities. Project knowledge cannot keep long time on account of the temporariness of projects. Ruuska and Vartiainen focus on the informal and semi-formal communities in project organizations. By means of the survey from eight types of virtual communities, the authors analyze the characteristic of KS communities from eight perspectives. Finally, the authors get the conclusion that "formality, leaning goals, practice based activities, coordination as enabling and enhancing contacts between members and the environment, organizational encouragement and focus on outcomes on personal, community, and organizational levels" are important elements of the communities [7].

Möller and Svahn examine the influence of ethnic culture on $\mathrm{KS}$ in different types of intercultural strategic nets. Their basic assumption is that the nature of the cultures involved and the type of business network both influence the knowledge-sharing barriers. This is investigated on the basis of two conceptual frameworks. The first is a framework for identifying four types of strategic nets - stable nets, dynamic nets and incremental nets as well as the requirements they impose for KS. Subsequently, a culture-classification scheme based on the individualism-collectivism and the vertical-horizontal dimensions is derived. The strategic net and cultural types are then combined in an intercultural business-net framework, and the opportunities for and barriers to KS are analyzed using examples representing Asian-European and Asian-North American joint ventures and networks [8].

Soekijad and Andriessen examine the conditions for successful KS and learning in inter-organizational alliances. In order to improve competitive advantage and business intelligence, organizations try to learn and develop knowledge in alliances. But how do they 'succeed' in these processes? The authors present several factors that are important for the 'successful' KS and learning in alliances where competing organizations co-operate. Their two case studies located in The Netherlands present some inter-personal conditions for 'successful' KS and learning in alliances [9].

Ryu et al. investigate the factors affecting physician's KS behavior within a hospital department by employing existing theories, namely the theory of reasoned action (TRA) and the theory of planned behavior (TPB). In the modified TPB model, subjective norms were found to have the strongest total effects on behavioral intentions to share knowledge of physicians through direct and indirect path by attitude. Attitude was found to be the second important factor influencing physicians' intentions. Perceived behavioral control was also found to affect the intention to share knowledge, though in a lesser degree than subjective norms or attitudes [10].

According to the compare and contrast of above technologies in Table 3, most researchers focus on how cultural issues influence KS performance, and other novel topics, such as the relationship between $\mathrm{KS}$ and its performance, the characteristics of KS communities and conditions for successful KS, are also researched. The research domains include commercial organizations, interorganizational alliances and some specific organizations, which generally tends to cover the primary organizational forms. These researches mostly use social research methods survey, case study and statistical analysis. The issues found by the researchers generally can be divided into two perspectives - subjective issues and objective issues. Subjective issues refer to personal motivation, behavior and so on; objective issues refer to organizational environment, rules, goals, mechanism and so on. Due to the difference of research domains, the results are different. Many researchers provide the solutions against these issues, in either subjective or objective aspect. In general, these researches support valuable knowledge to $\mathrm{KS}$ in own domain, which has huge economic value. However, the research result base on limited amount of case studies and theories or relatively survey small scope, which can be critically considered as ex parte on the other hand.

TABLE 1

COMPARE AND CONTRAST OF THE CULTURAL RESEARCHES ON KNOWLEDGE SHARING (PART 1) 


\begin{tabular}{|c|c|c|c|}
\hline Taptis & 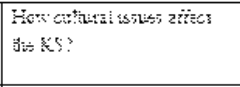 & 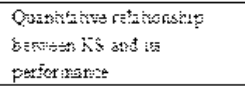 & 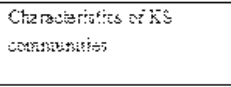 \\
\hline Dofzain & 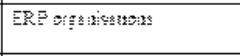 & 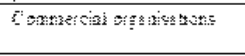 & Projent organisat ons \\
\hline $\begin{array}{l}\text { Rasery ih } \\
\text { matgots } \\
\text { frgaes }\end{array}$ & 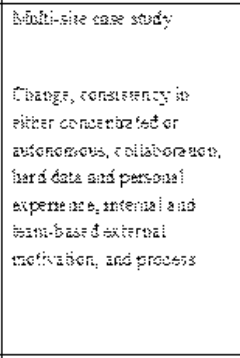 & 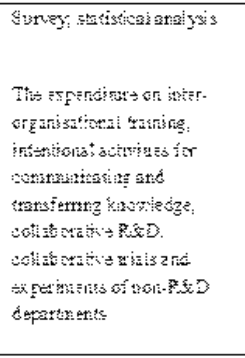 & 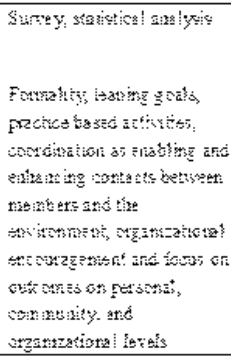 \\
\hline $\begin{array}{l}\text { Sehations in } \\
\text { possibles }\end{array}$ & 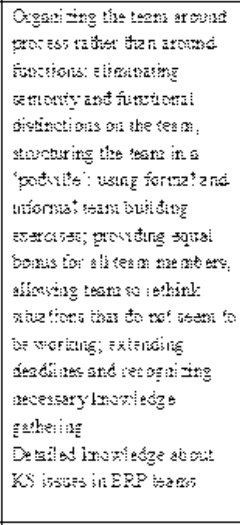 & 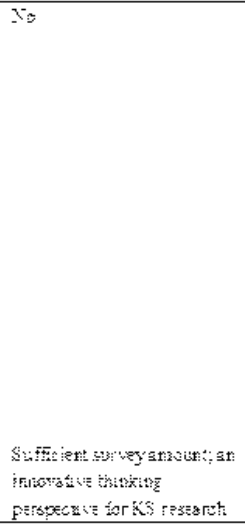 & 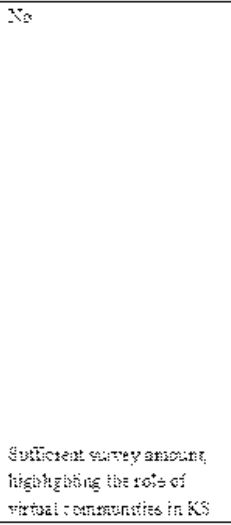 \\
\hline 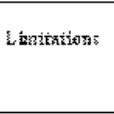 & 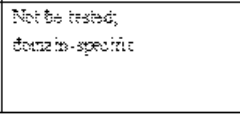 & 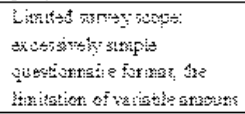 & 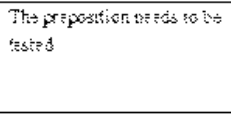 \\
\hline
\end{tabular}

TABLE 2

COMPARE AND CONTRAST OF THE CULTURAL RESEARCHES ON KNOWLEDGE

\begin{tabular}{|c|c|c|c|}
\hline \\
\hline Dowaiม & 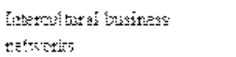 & 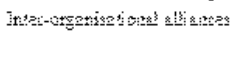 & 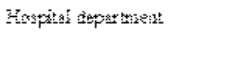 \\
\hline & 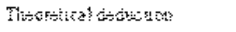 & 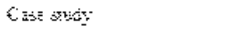 & 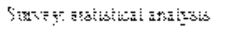 \\
\hline $\operatorname{liszaez}$ & 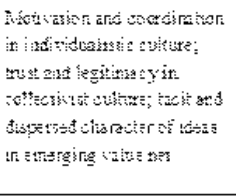 & 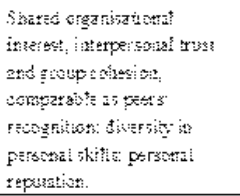 & 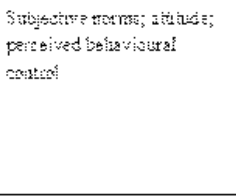 \\
\hline $\begin{array}{l}\text { Selations if } \\
\text { porctions? }\end{array}$ & 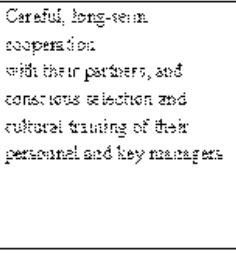 & $x_{0}$ & 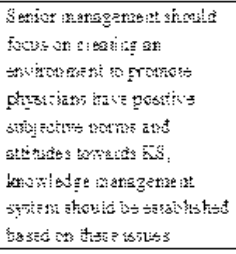 \\
\hline 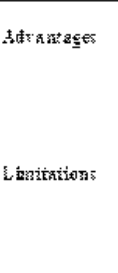 & 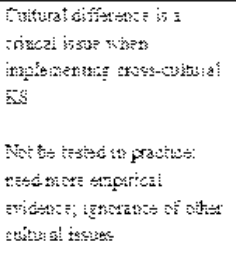 & 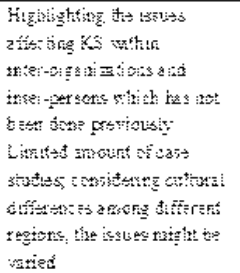 & 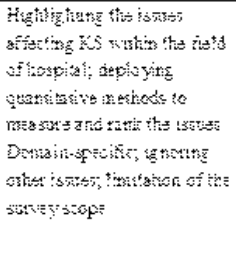 \\
\hline
\end{tabular}

\section{CONCLUSION AND FUTURE WORKS}

In this paper, we review the contemporary primary research outcomes in the field of KS. In this paper, we review the contemporary primary research outcomes in the field of KS. From cultural perspective, we introduce the KS research situation, and compare the characteristics of these researches. For cultural KS researches, we introduce six articles mainly about the cultural issues which influence $\mathrm{KS}$ and its performance. These papers basically cover the primary organizational forms - single organizations, network organizations and organizational alliances. By means of a great number of surveys, statistical analysis and case studies, the issues which affect KS can be divided into subjective issues and objective issues. Although the issues have certain limitations, these researches provide valuable $\mathrm{KS}$ knowledge to organizational management

In general, these literatures represent the situation of the current KS researches. By critically analysing the papers, it is observed that the positive changes are occurring in $\mathrm{KS}$ researches, which tends to be multidisciplinary, cross-field, more universal and closer to our life, with highest research potential and commercial values. Thus, we believe that the future KS researches can recognize the issues stated in our paper and self-improvement according to the general trend we present.

\section{ACKNOWLEDGMENT}

We would like to express our gratitude to the assistance of DEBII and School of Information Systems in Curtin University of Technology.

\section{REFERENCES}

[1] D. Bobrow \& J. Whalen (2002) Community Knowledge Sharing in Practice: The Eureka Story. Joumal of the Society for organization learning and MTT Press, 4(2).

[2] M. C. Jones, M. Cline \& S. Ryan (2006) Exploring Knowledge Sharing in ERP Impl ementation: an Organizational Culture Framework. Decision Support Systems, 41(2): 411434.

[3] J. Cummings (2003) Knowledge Sharing: A Review of the Literature.

[4] M. Soekijad \& E. Andri essen (2003) Conditions for Knowledge Sharing in Competitive Alliances. European Management Joumal, 21(5): 578587.

[5] S. Ryu, S. H. Ho \& I. Han (2003) Knowledge Sharing Behaviour of Physicians in Hospital s. Expert Systems with Applications, 25(1):113-122.

[6] R. Du, S. Ai \& Y. Ren (2005) Relationship between Knowledge Sharing and Performance: a Survey in Xi'an, China, Expert Systems with Applications.

[7] I. Ruuska \& M. Vartiainen (2005) Characteristics of Knowledge Sharing Communities in Project Organizations. International Joumal of Project Management, 23(5): 374-379.

[8] K. Möller \& S. Svahn (2004) Crossing East-West Boundaries: Knowledge Sharing in Intercultural Business Networks. Industrial Marketing Management, 33(3): 219-228.

[9] M. Soekijad \& E. Andriessen (2003) Conditions for Knowledge Sharing in Competitive Alliances. European Management Joumal, 21(5): $578-$ 587.

[10] S. Ryu, S. H. Ho \& I. Han (2003) Knowledge Sharing Behaviour of Physicians in Hospitals. Expert Systems with Applications, 25(1): 113122 . 